\title{
Clasificación de prácticas de educción de requisitos en desarrollos ágiles: un mapeo sistemático
}

\author{
Classification of requirements elicitation practices in agile \\ developments: a systematic mapping \\ Dante Carrizo $^{1 *} \quad$ Jorge Rojas ${ }^{1}$ \\ Recibido 17 de diciembre de 2015, aceptado 7 de marzo de 2016 \\ Received: December 17, 2015 Accepted: March 7, 2016
}

\begin{abstract}
RESUMEN
Los métodos de desarrollos ágiles priorizan los requisitos para obtener entregas incrementales en funcionalidad. Por lo que la educción de estos requisitos debe seleccionar estas funcionalidades principales y especificarlas eficientemente. No obstante, aún no existen catastros de prácticas que orienten en este sentido. Este trabajo pretende identificar la mayor cantidad de prácticas de educción en desarrollos ágiles de la literatura científica y clasificarlas según el foco principal de cada práctica. Para esto se lleva a cabo un mapeo sistemático sobre las principales bases de datos del área. Los resultados obtenidos dan cuenta de un insuficiente reporte de guías de educción para los desarrolladores ágiles. Los estudios primarios son principalmente teóricos, esto quiere decir que las prácticas de educción de requisitos en métodos ágiles aún requieren más validación empírica para ser aceptadas. Es necesaria la definición de ontologías, respecto a educción en desarrollos ágiles, que faciliten la identificación de prácticas útiles para la obtención de productos exitosos.
\end{abstract}

Palabras clave: Requisitos software, métodos ágiles, educción, prácticas, mapeo sistemático.

\begin{abstract}
Agile development methods prioritize the requirements for incremental delivery functionality. So the elicitation of these requirements must select these main features and specify them efficiently. However, there are still no registers that drive practices in this way. This work aims to identify as many elicitation practices in agile developments of scientific literature and classify them according to the main focus of each guideline. For this, it carries out a systematic mapping over main databases in this area. The results obtained show insufficient reporting guidelines for agile developers. Primary studies are mainly theoretical, this means that the requirements for elicitation practices in agile methods still require further empirical validation to be accepted. The definition of ontology is absolutely necessary, regarding elicitation in agile developments, to facilitate the identification of useful practices for obtaining successful products.
\end{abstract}

Keywords: Software requirements, agile methods, elicitation, practices, systematic mapping.

1 Departamento de Ingeniería Informática y Ciencias de la Computación, Universidad de Atacama. Av. Copayapu 485. Copiapó, Chile.E-mail: dante.carrizo@uda.cl; jorge.rojas@alumnos.uda.cl

* Autor de correspondencia 


\section{INTRODUCCIÓN}

El alto desarrollo tecnológico, el cambio en la preferencia de los stakeholders y las restricciones de tiempo y costos, generan una toma y especificación de requisitos inapropiadas. Es por ello que las metodologías ágiles buscan hacer frente a estos retos evitando el compromiso prematuro y discutiendo los requisitos de manera iterativa [1].

Ya sea de un gran proyecto de software industrial o un proyecto de software para un micronegocio, se requiere un proceso de requisitos riguroso que evite errores de omisión [2]. El análisis de requisitos es un proceso difícil, ya que los diferentes stakeholders tienen visiones, a veces contradictorias, y pueden expresarlas de maneras muy diferentes.

Esto hace que los desarrolladores no entiendan los requisitos del sistema y puedan obtener productos finales defectuosos [3]. Para evitar esto, sería necesario contar con guías para conducir la educción de requisitos en modelos de desarrollos ágiles que faciliten tanto el descubrimiento como la definición de estos requisitos.

No obstante, cabe notar que todavía no existen publicaciones que recopilen prácticas de educción de requerimientos en métodos ágiles y entreguen una caracterización de las prácticas encontradas.

Este artículo está divido en cinco secciones. La segunda sección describe la metodología utilizada para el mapeo sistemático. La tercera sección presenta el análisis realizado para la selección de los trabajos primarios del estudio. La cuarta sección presenta los resultados obtenidos y, finalmente, la quinta sección presenta las conclusiones y perspectivas de trabajos futuros.

\section{METODOLOGÍA}

Para el desarrollo de este estudio utilizamos la metodología que nos permite obtener una mayor evidencia de información sobre las prácticas existentes en el proceso de educción de requisitos de software de desarrollo ágil.

Este estudio requiere una revisión menos exhaustiva que una revisión sistemática de literatura que pretende generar un cuerpo de conocimiento en un área. En este caso, se desea obtener una visión amplia del campo científico, focos de atención y tendencias de los investigadores.

Por este motivo hemos considerado un mapeo sistemático de la literatura con una modificación en los procesos propuestos por $[4,5]$. Los nuevos procesos son: definición de pregunta de investigación, alcance de la revisión -donde se definen las bases de datos de publicaciones científicas y cadena de búsqueda-, definición de los criterios de inclusión y exclusión, conducta de búsqueda, selección de trabajos primarios, definición de criterios de análisis, esquema de caracterización; y el análisis de resultados.

La metodología seguida se presenta en la Figura 1.

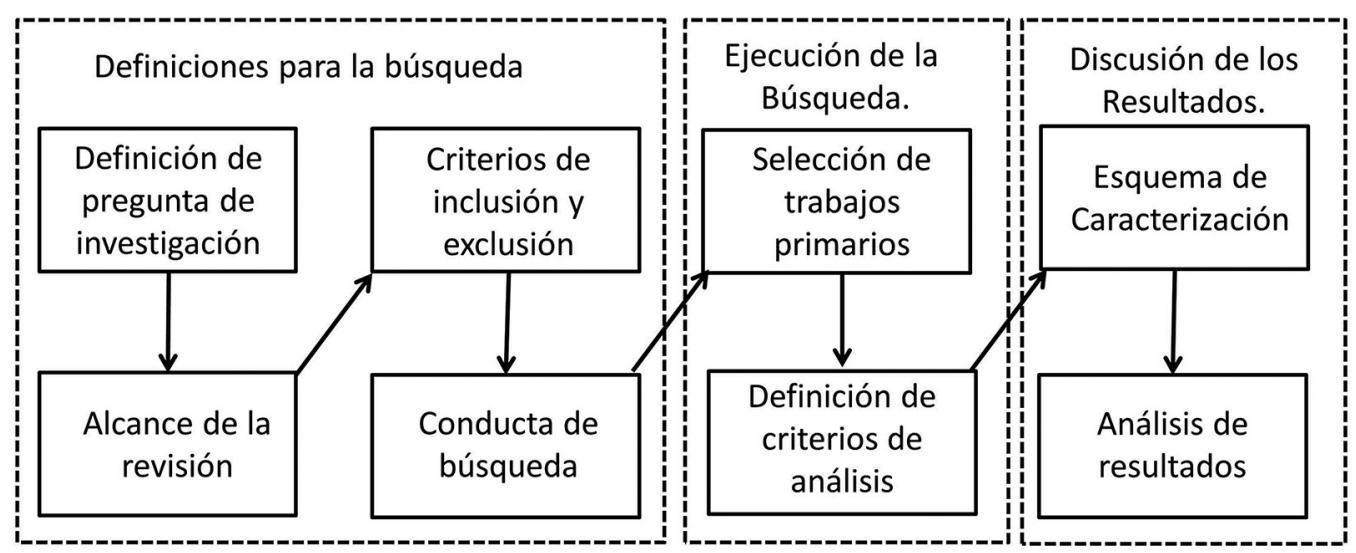

Figura 1. Procesos del mapeo sistemático. 


\section{DEFINICIONES PARA LA BÚSQUEDA}

\section{Pregunta de investigación}

Debido a que aún no existen directrices para efectuar la tarea de requisitos con métodos ágiles de forma consensuada, es que este este estudio intenta responder a la siguiente pregunta de investigación:

RQ1: ¿Qué prácticas en el proceso de educción de requisitos de software de desarrollos ágiles han sido reportadas en la literatura científica?

\section{Alcance de revisión}

A pesar de que existe un extenso listado de bases de datos de publicaciones relacionadas con informática, en este estudio nos hemos focalizado en las principales fuentes de investigaciones en el área de la ingeniería de software.

Las bases de datos apropiadas para encontrar la mayor cantidad de publicaciones relacionadas son: IEEEXPLORE, ACM DL, SCOPUS, y WEB OF SCIENCE. También se realizaron búsquedas oportunistas, referencias de artículos relacionados, y otros artículos ya identificados.

El período de búsqueda incluye publicaciones desde los inicios del manifiesto ágil [6] en el año 2001 hasta diciembre del 2014.

La cadena de búsqueda utilizada en las bases de datos de publicaciones es:

(requirements AND elicitation AND agile)

Esta cadena se ajustó a los propios formatos de cada base de datos.

\section{Criterios de inclusión y exclusión}

Para la selección de estudios se consideraron los siguientes criterios de inclusión/exclusión:

- Toda publicación científica es elegible para su inclusión en el estudio si tiene relación con requisitos de software y en donde se propusieran, discutieran o evaluaran metodologías sobre el desarrollo de software ágil.

- Se incluyeron tanto estudios cualitativos y cuantitativos realizados por estudiantes como profesionales del software.

- Solo estudios en inglés fueron incluidos.
- Se excluyeron todos aquellos estudios en los que su enfoque principal se alejara del desarrollo de software ágil.

- Los artículos sin diseño de investigación, basados en opiniones de expertos y sin una pregunta de investigación también fueron excluidos.

\section{Conducta de búsqueda}

Para llegar a identificar los estudios primarios se realizaron los siguientes filtros de revisión:

$1^{\text {er }}$ filtro:

- Revisión de Resumen o Abstract.

$2^{\text {do }}$ filtro:

- Las publicaciones que pasaron el filtro anterior fueron sometidas a una lectura y análisis completo de su contenido.

\section{EJECUCIÓN DE LA BÚSQUEDA}

\section{Selección de trabajos primarios}

Los resultados de la búsqueda pueden ser observados en la Figura 2. Después de aplicar el primer y segundo filtro fueron seleccionadas 13 publicaciones primarias.

Es importante destacar que tanto como en la búsqueda oportunista como en las bases de datos mencionadas, no se registraron publicaciones similares a la búsqueda de prácticas de educción de requisitos en el desarrollo de software ágil.

\section{Definición de criterios de análisis}

Para analizar cada trabajo encontrado se ha definido un conjunto de criterios que sirven como esquema de clasificación para obtener una vista focalizada de los estudios.

Tipo de estudio: Entendiendo como tipo de estudio empírico el que muestra investigación de campo en la educción de requisitos, ya sean estudios de casos, surveys o experimentos controlados. En caso contrario se entenderá como un tipo de estudio teórico.

Tipo de metodología: Cualquier práctica encontrada en la que no se especifica en qué tipo de metodología ágil de software es aplicada, o se deja abierta la posibilidad de ser aplicada en cualquier metodología ágil, se entenderá como general. En el caso que se especifique la metodología que es aplicada, como 


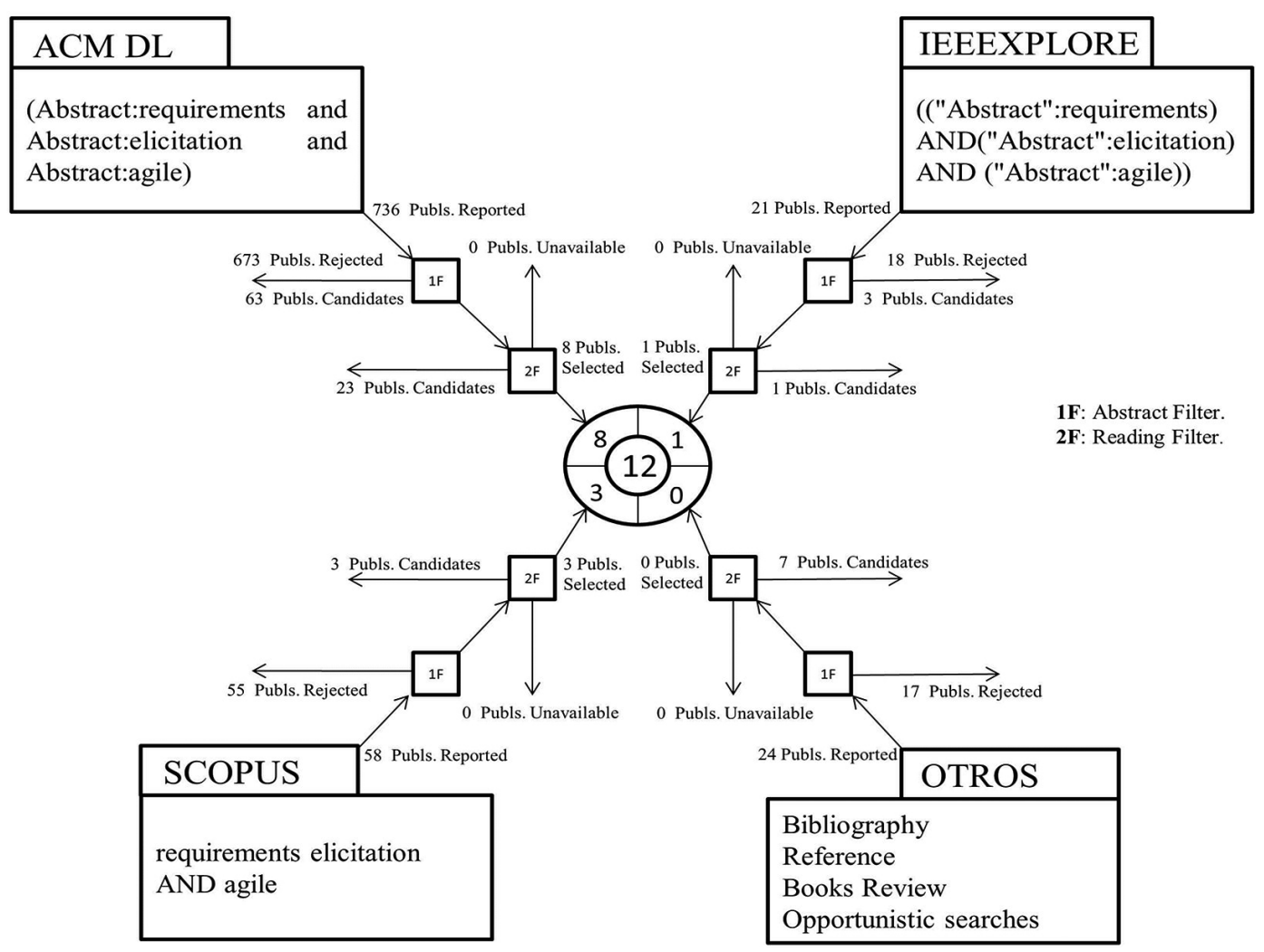

Figura 2. Gráfico de búsqueda de estudios primarios en formato DCM.

por ejemplo XP, SCRUM, FDD, etc., será clasificada como particular.

Foco: Siendo el cuerpo principal hacia donde está dirigida cada práctica se creó la siguiente clasificación.

- Proceso: Toda práctica en la que es necesaria su aplicación en una fase de transformación de requisitos.

- Herramienta: Cualquier instrumento que facilite la educción de requisitos.

- Ambiente: Entendiendo como todo medio físico qué interactúa en el proceso de educción de requisitos.

- Técnica: Conjunto de procedimientos que tienen como objetivo definir requisitos.

- Personas: Características o roles que debe adquirir un individuo en un proceso de educción de requisitos.

- Negocio: Prácticas asociadas al modelado de procesos de negocios.

\section{DISCUSIÓN DE LOS RESULTADOS}

\section{Análisis de estudios primarios}

A continuación en la Tabla 1 se pueden ver los trabajos encontrados con información sobre el tipo de estudio que se realizó, el objetivo principal de la práctica encontrada y en qué tipo de metodología ágil son aplicados. Es importante destacar que la mayoría de los trabajos primarios identificados son superiores al año 2007.

En la Figura 3 se muestra una visión más completa del mapeo sistemático en forma de un gráfico burbuja. En él, se clasifican los estudios primarios de acuerdo a los criterios de análisis ya mencionados. Además, se puede apreciar que aún no hay una clara tendencia sobre qué tipo de prácticas de educción de requisitos en métodos ágiles se están utilizando y desarrollando, pero lo que sí se puede observar es que todavía falta por poner en práctica el trabajo teórico realizado por los investigadores. 
Tabla 1. Trabajos primarios identificados.

\begin{tabular}{|l|c|c|c|l|l|}
\hline \multicolumn{1}{|c|}{ Autores } & Año & Ref. & Tipo de estudio & \multicolumn{1}{|c|}{ Foco } & Metodología \\
\hline L. Cao and B. Ramesh & 2008 & {$[8]$} & Empírico & Personas & Particular \\
\hline L. Cao and B. Ramesh & 2008 & {$[8]$} & Empírico & Proceso & Particular \\
\hline $\begin{array}{l}\text { R. Macasaet, L. Chung, J.L. Garrido, M. } \\
\text { Noguera and M.L. Rodríguez }\end{array}$ & 2011 & {$[9]$} & Teórico & Negocio & General \\
\hline $\begin{array}{l}\text { R. Macasaet, L. Chung, J.L. Garrido, M. } \\
\text { Noguera and M.L. Rodríguez }\end{array}$ & 2011 & {$[9]$} & Teórico & Proceso & General \\
\hline $\begin{array}{l}\text { M.G. Chand, a. A. Rao, K.N. Reddy and J.K. } \\
\text { Kumar }\end{array}$ & 2010 & {$[3]$} & Teórico & Técnica & Particular \\
\hline J.S. de Oliveira Neto and L.V.L. Filgueiras & 2008 & {$[10]$} & Teórico & Herramienta & General \\
\hline $\begin{array}{l}\text { C.D. Nguyen, E. Gallagher, A. Read and G.J. } \\
\text { De Vreede }\end{array}$ & 2009 & {$[11]$} & Teórico & Técnica & General \\
\hline R. Akbar, M. Haris and M. Naeem & 2008 & {$[12]$} & Teórico & Personas & General \\
\hline D. Connolly, F. Keenan and B. Ryder & 2008 & {$[13]$} & Teórico & Herramienta & General \\
\hline S. Kelly & 2010 & {$[14]$} & Empírico & Ambiente & Particular \\
\hline M.C. Leonardi, J. Cesar and P. Leite & 2002 & {$[15]$} & Teórico & Técnica & Particular \\
\hline M. Maguire & 2013 & {$[16]$} & Teórico & Técnica & General \\
\hline A. Singhal. & 2011 & {$[17]$} & Teórico & Técnica & General \\
\hline A.N. Bowers, R. S. Sangwan and C.J. Neill & 2007 & {$[18]$} & Empírico & Personas & General \\
\hline
\end{tabular}

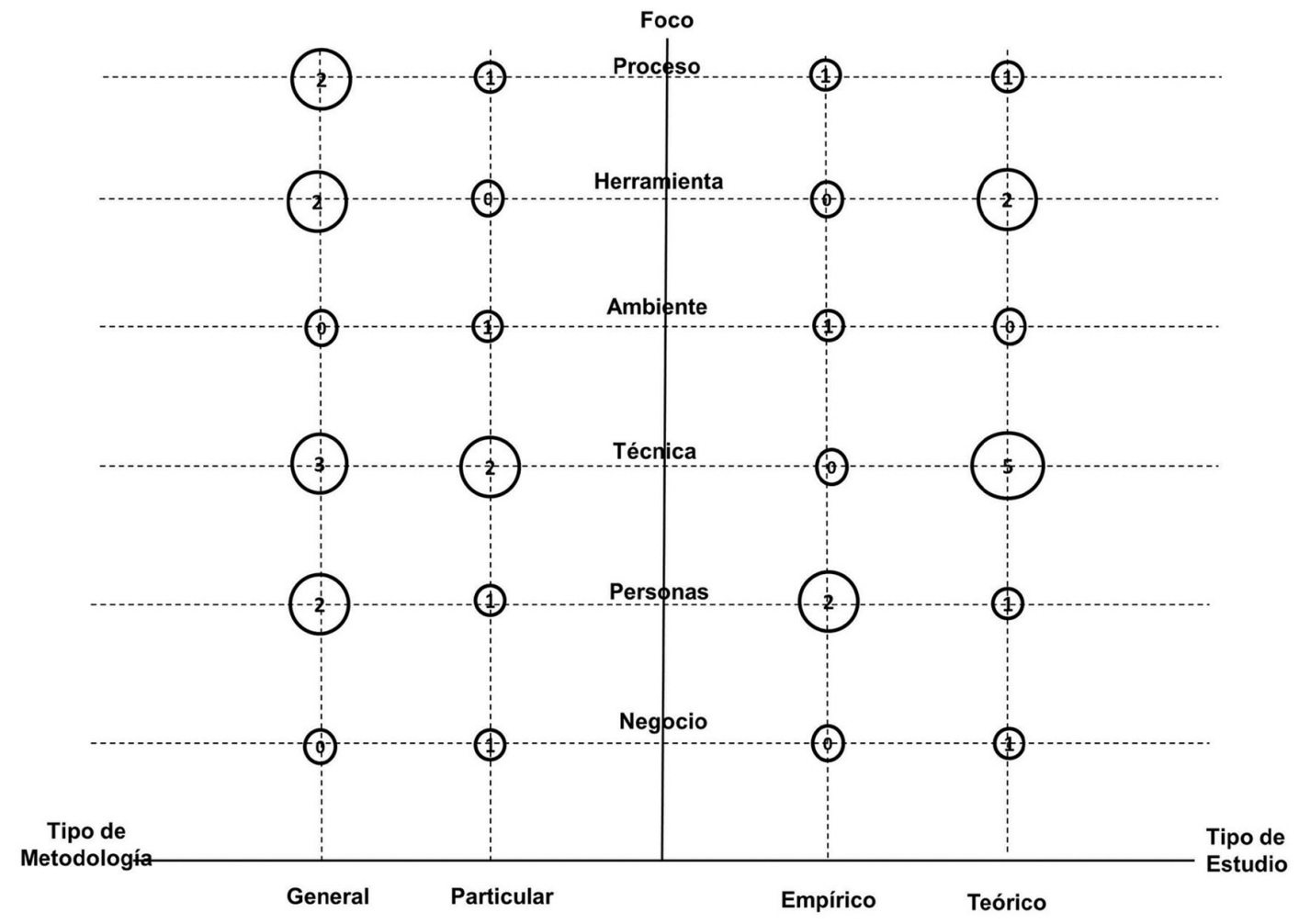

Figura 3. Gráfico de burbuja mapeo sistemático. 


\section{Discusión por criterios}

En la Tabla 2 se puede apreciar todas las prácticas encontradas en los estudios primarios como respuesta a nuestra pregunta de investigación (RQ1).

A continuación se presenta una discusión por los criterios definidos en el esquema de caracterización.

\section{Tipos de estudio}

En los trabajos primarios encontrados se puede observar que la mayoría fueron realizados desde el
2007 en adelante. También puede verse que varios de ellos son desarrollos teóricos.

Esto puede implicar que las prácticas de educción de requisitos en métodos ágiles aún requieren más validación del medio para ser aceptadas.

\section{Tipo de metodología}

En algunos casos, las prácticas encontradas se aplicaron para un tipo de metodología en particular. Como por ejemplo en [8], el foco

Tabla 2. Prácticas de educción de requisitos de educción de requisitos en desarrollo ágil de software.

\begin{tabular}{|c|c|c|c|c|}
\hline $\mathbf{N}^{0}$ & Título & Autores & Prácticas & Foco \\
\hline \multirow[b]{2}{*}{1} & \multirow[b]{2}{*}{$\begin{array}{l}\text { Agile Requirements } \\
\text { Engineering Practices, } \\
\text { An Empirical Study. }\end{array}$} & \multirow[b]{2}{*}{ Cao, L. \& Ramesh, B. } & $\begin{array}{l}\text { La mayoría de las organizaciones pretenden transferir } \\
\text { eficazmente las ideas del cliente al equipo de } \\
\text { desarrollo, para ello, utilizan técnicas sencillas como } \\
\text { las historias de usuario para definir los requisitos } \\
\text { de alto nivel, prefiriendo la comunicación cara a } \\
\text { cara sobre las especificaciones escritas. }\end{array}$ & Personas \\
\hline & & & $\begin{array}{l}\text { Al inicio de cada ciclo, en RE ágil el cliente se } \\
\text { reúne con el equipo de desarrollo para proporcionar } \\
\text { información detallada sobre un conjunto de } \\
\text { características que deben implementarse. Durante } \\
\text { este proceso, los requisitos se discuten en un mayor } \\
\text { nivel de detalle. Además, RE suele estar íntimamente } \\
\text { vinculada con el diseño. Esta actividad a menudo } \\
\text { resulta en un set de requerimientos refinados y } \\
\text { un diseño preliminar, e incluso a veces un plan } \\
\text { de implementación, ninguno de los cuales se } \\
\text { especifica formalmente. }\end{array}$ & Proceso \\
\hline \multirow[t]{2}{*}{2} & \multirow{2}{*}{$\begin{array}{l}\text { An Agile Requirements } \\
\text { Elicitation Approach } \\
\text { based on NFRs and } \\
\text { Business Process Models } \\
\text { for Micro-Businesses }\end{array}$} & \multirow{2}{*}{$\begin{array}{l}\text { Macasaet, R., Chung, } \\
\text { L., Garrido, J.L., } \\
\text { Noguera, M. \& } \\
\text { Rodríguez, M.L. }\end{array}$} & $\begin{array}{l}\text { Para obtener y definir requisitos adecuados, es } \\
\text { importante que el propietario de la microempresa } \\
\text { enumere los objetivos del negocio y diagrame } \\
\text { los procesos de negocios, para que junto con el } \\
\text { desarrollador analicen estos diagramas y encuentren } \\
\text { patrones similares de negocios existentes que sean } \\
\text { de utilidad. }\end{array}$ & Negocio \\
\hline & & & $\begin{array}{l}\text { Los requerimientos no funcionales deben ser } \\
\text { objetivos y tiene que haber algún proceso donde } \\
\text { sean probados. Cuando los requisitos funcionales } \\
\text { y no funcionales están definidos claramente, el } \\
\text { desarrollador de software solo tiene que satisfacerlos. }\end{array}$ & Proceso \\
\hline 3 & $\begin{array}{l}\text { An approach to } \\
\text { requirements elicitation } \\
\text { and analysis using goal }\end{array}$ & $\begin{array}{l}\text { Chand, M.G., Reddy, } \\
\text { K.N., Rao, A.A. \& } \\
\text { Kumar, J.K. }\end{array}$ & $\begin{array}{l}\text { Para una mejor comprensión de sistemas más } \\
\text { complejos se debe obtener múltiples vistas de los } \\
\text { distintos stakeholders. Para ello se debe utilizar un } \\
\text { conjunto de palabras claves en la identificación de } \\
\text { requerimientos de entrada, del sistema y de salida. }\end{array}$ & Técnicas \\
\hline 4 & $\begin{array}{l}\text { Crossmedia Application } \\
\text { Design Exploring Linear } \\
\text { and Non-linear Narrative } \\
\text { Abilities }\end{array}$ & $\begin{array}{l}\text { de Oliveira Neto, J.S. \& } \\
\text { Filgueiras, L.V. (2008, } \\
\text { September). }\end{array}$ & $\begin{array}{l}\text { En la fase de educción de requerimientos se podrían } \\
\text { utilizar herramientas crossmedia que facilite el uso } \\
\text { de artefactos narrativos, escenarios, mapas, esquemas } \\
\text { y guiones gráficos, para aclarar restricciones de los } \\
\text { medios, limitaciones de accesibilidad del usuario } \\
\text { y enfatizar algunos puntos cruciales para todo el } \\
\text { proceso de desarrollo del sistema. }\end{array}$ & Herramienta \\
\hline
\end{tabular}




\begin{tabular}{|c|c|c|c|c|}
\hline $\mathbf{N}^{0}$ & Título & Autores & Prácticas & Foco \\
\hline 5 & $\begin{array}{l}\text { Generating User Stories } \\
\text { in Groups }\end{array}$ & $\begin{array}{l}\text { Read, A., Gallagher, } \\
\text { E., Nguyen, C. \& de } \\
\text { Vreede, G.J. }\end{array}$ & $\begin{array}{l}\text { Permitir que los stakeholders generen historias en } \\
\text { grupos para facilitar el flujo de información valiosa } \\
\text { y que no olviden información como a veces sucede } \\
\text { en historias de usuarios. Además permite que un } \\
\text { problema pueda ser visto desde varias perspectivas } \\
\text { y el conocimiento de un stakeholder puede ser } \\
\text { ampliado por otro. }\end{array}$ & Técnicas \\
\hline 6 & $\begin{array}{l}\text { Requirement gathering } \\
\text { and tracking process for } \\
\text { distributed agile based } \\
\text { development }\end{array}$ & $\begin{array}{l}\text { Akbar, R., Haris, M. \& } \\
\text { Naeem, M. }\end{array}$ & $\begin{array}{l}\text { En grandes proyectos de software, es necesario } \\
\text { contar con un equipo adecuado para un desarrollo } \\
\text { ágil distribuido. Este equipo puede estar conformado } \\
\text { por un gerente de proyecto, un programador senior, } \\
\text { y una persona de control de calidad. Estos son los } \\
\text { encargados verificar y gestionar los cambios de las } \\
\text { necesidades de los stakeholders, además de facilitar } \\
\text { la comunicación directa con ellos. }\end{array}$ & Personas \\
\hline 7 & $\begin{array}{l}\text { Tag Oriented Agile } \\
\text { Requirements Iden- } \\
\text { tification. }\end{array}$ & $\begin{array}{l}\text { Connolly, D., Keenan, } \\
\text { F. \& Ryder, B. }\end{array}$ & $\begin{array}{l}\text { El uso de herramientas bookmarklet facilita la } \\
\text { vinculación del prototipado con las historias de } \\
\text { usuarios. Esto permite que potenciales requisitos } \\
\text { sean identificados rápidamente y que las historias } \\
\text { de usuarios sean creadas y documentadas. }\end{array}$ & Herramienta \\
\hline 8 & $\begin{array}{l}\text { Towards an Evolutionary } \\
\text { Framework for Agile } \\
\text { Requirements Elicitation }\end{array}$ & Kelly, S. & $\begin{array}{l}\text { Proporcionar un espacio abierto donde los } \\
\text { stakeholders puedan discutir, acordar temas, } \\
\text { desarrollar escenarios, etc. Esto crea un ambiente } \\
\text { de aprendizaje interactivo, donde desarrolladores } \\
\text { y stakeholders intercambian conocimientos y } \\
\text { habilidades para avanzar hacia una solución de } \\
\text { manera colaborativa. }\end{array}$ & Ambiente \\
\hline 9 & $\begin{array}{l}\text { Using Business Rules in } \\
\text { EXtreme Requirements }\end{array}$ & $\begin{array}{l}\text { Leonardi, M.C. \& do } \\
\text { Prado Leite, J.C.S. }\end{array}$ & $\begin{array}{l}\text { Para mejorar la comunicación con los stakeholders, } \\
\text { es necesario desarrollar un vocabulario y una } \\
\text { semántica que unifique el lenguaje. Esto facilita } \\
\text { la comprensión del problema, el proceso de } \\
\text { construcción de escenarios, reglas de negocio y } \\
\text { ayuda en su descripción. }\end{array}$ & Técnicas \\
\hline 10 & $\begin{array}{l}\text { Using Human Factors } \\
\text { Standards to Support } \\
\text { User Experience and } \\
\text { Agile Design }\end{array}$ & Maguire, M. & $\begin{array}{l}\text { La trayectoria de usuarios, es decir, viajes que llevan } \\
\text { al usuario de una etapa a otra, tiene como propósito } \\
\text { tanto guiar paso a paso a un usuario hasta alcanzar } \\
\text { su objetivo, creando un viaje ideal y mejorando su } \\
\text { experiencia, como permitir a los desarrolladores } \\
\text { definir los requisitos no funcionales. }\end{array}$ & Técnicas \\
\hline 11 & $\begin{array}{l}\text { Development of Agile } \\
\text { Security Framework } \\
\text { Using a Hybrid Techni- } \\
\text { que for Requirements } \\
\text { Elicitation }\end{array}$ & Singhal, A. & $\begin{array}{l}\text { La utilización en conjunto de historias de intrusión } \\
\text { (historias realizadas por un vulnerador de sistemas) } \\
\text { y árboles de ataque (diagramas de árbol que detallan } \\
\text { las diferentes formas de alcanzar un objetivo } \\
\text { de ataque) en la educción de requerimientos de } \\
\text { seguridad permite agilizar el proceso de generar } \\
\text { estrategias para evitar amenazas y comprender el } \\
\text { cómo se efectúan ataques al sistema. }\end{array}$ & Técnicas \\
\hline 12 & $\begin{array}{l}\text { Adoption of XP Practices } \\
\text { in the Industry - A Survey }\end{array}$ & $\begin{array}{l}\text { Alexia N. Bowers, } \\
\text { Raghvinder S. Sangwan } \\
\text { and J. Neill. }\end{array}$ & $\begin{array}{l}\text { El equipo puede tener una persona en el lugar, que } \\
\text { puede elegir, priorizar y verificar los requisitos del } \\
\text { sistema, idealmente, un usuario final del sistema. }\end{array}$ & Personas \\
\hline
\end{tabular}

principal es preferir la comunicación cara a cara sobre las especificaciones escritas. Esta práctica fue utilizada en XP, pero puede ser aplicada en cualquier otra metodología en donde se facilita la interacción con los stakeholders. Es decir, prácticas que fueron ejecutadas en un solo tipo de metodología ágil, podrían ser utilizadas en otros tipos de metodologías. 


\section{Foco}

Con respecto al foco de tipo proceso, lograr que el cliente se reúna con el equipo de desarrolladores para así lograr un refinamiento y validación de requisitos permite que los programadores solo se dediquen a satisfacerlos.

En muchas ocasiones los clientes no están claros desde el principio acerca de sus necesidades y están dispuestos a explorar nuevas formas en que el sistema en evolución puede ayudar a sus objetivos del negocio. [8] Esto genera una relación más satisfactoria con el cliente.

En el foco tipo herramienta, el uso intensivo de tecnologías puede ser un obstáculo en vez de un beneficio, es por ello que la utilización de herramientas que permitan la integración de diferentes maneras de entregar un mismo mensaje facilita la comprensión por parte de los desarrolladores.

Referente al foco tipo ambiente, proveer de un espacio abierto donde los stakeholders y desarrolladores puedan desarrollar escenarios, llegar a acuerdos, etc. No solo crea un ambiente de desarrollo interactivo donde stakeholders y desarrolladores intercambian conocimientos. También facilita la negociación efectiva.

Acerca del foco tipo técnica, la mayor cantidad de prácticas encontradas se centran en técnicas que buscan obtener requerimientos bien definidos y ampliar el conocimiento tanto del stakeholder como desarrolladores. De igual forma es aconsejable: tener varias vistas a un problema en particular, unificar el lenguaje, guiar al stakeholder a un viaje ideal y generar nuevas estrategias.

Con respecto al foco tipo personas, la educción de requisitos de software ágil en grandes proyectos de software los actores deben tener bien claro el rol que ellos cumplirán. Destacando como sobresaliente la comunicación entre stakeholders y desarrolladores. También la presencia de tener un usuario final del sistema que pueda priorizar $\mathrm{y}$ verificar requisitos.

En el foco tipo negocio, los objetivos y procesos de negocios, siempre deben estar bien definidos para que los desarrolladores encuentren similitudes entre diagramas y patrones de negocio existentes.

\section{CONCLUSIONES}

Descubrir y definir adecuadamente los requisitos es una parte fundamental de la ingeniería de software. Junto con los métodos ágiles han demostrado varias ventajas, tales como facilitar la comprensión del dominio del problema, generar entornos de aprendizajes tanto para stakeholders y desarrolladores, detectar errores en etapas tempranas, generar documentación mínima, etcétera.

La poca documentación con respecto a las prácticas de educción ágil originó que para rescatar algunas prácticas se necesitó aplicar un mayor nivel de conceptualización. Lo que conlleva a una falta de formalización de las propuestas de los investigadores para que estas prácticas sean adoptadas por los desarrolladores de software.

Como trabajo futuro los autores plantean la definición de una ontología, respecto a los desarrollos ágiles, que facilite la identificación de prácticas necesarias para la obtención de productos exitosos.

\section{REFERENCIAS}

[1] N.A. Ernst, A. Borgida, I.J. Jureta and J. Mylopoulos. "Agile requirements engineering via paraconsistent reasoning". Information Sysems. Vol. 43, pp. 100-116. 2014.

[2] T. Marew, J. Kim and D.H. Bae. "Systematic Functional Decomposition in a Product Line Using Aspect-Oriented Software Development: a Case Study". International Journal Software Enginering and Knowledge Enginering. Vol. $17 \mathrm{~N}^{\mathrm{o}}$ 1, pp. 33-55. 2007.

[3] M.G. Chand, A.A. Rao, K.N. Reddy and J. K. Kumar. "An approach to requirements elicitation and analysis using goal". Proceedings 2nd International Conference Software Technological Enginering. Vol. 1, pp. 218-221. 2010.

[4] D. Carrizo Moreno. "Atributos contextuales influyentes en el proceso de educción de requisitos: una exhaustiva revisión de literatura”. Ingeniare, Revista chilena de ingeniería. Vol. 23, pp. 208-218. 2015.

[5] K. Petersen, R. Feldt, S. Mujtaba and M. Mattsson. "Systematic mapping studies in software engineering". In 12th International 
Conference on Evaluation and Assessment in Software Engineering. Vol. $17 \mathrm{~N}^{\circ} 1.2008$.

[6] J.F. Urquiza, A. Martínez y G. Ibarguengoitía. "Las Metodologías Ágiles y las Arquitecturas de Software". Coloquio Nacional de Investigación en Ingeniería de Software y Vinculación Academia-Industria. 2010.

[7] N. Baloian, J.A. Pino, C. Reveco and G. Zurita. "Mobile collaboration for business process elicitation from an agile development methodology viewpoint". Proceedings IEEE 10th International Conference E-bussiness Enginering. pp. 306-311. 2013.

[8] L. Cao and B. Ramesh. "Agile requirements engineering practices: An empirical study". IEEE Software. Vol. $25 \mathrm{~N}^{\circ}$ 1, pp. 60-67. 2008.

[9] R. Macasaet, L. Chung, J.L. Garrido, M. Noguera and M. L. Rodríguez. "An Agile Requirements Elicitation Approach Based on \{NFRs $\}$ and Business Process Models for Micro-businesses". Proc. eedings 12th International Conference Product. Focused Software Development Process Improvement, pp. 50-56. 2011.

[10] J. S. de Oliveira Neto and L. V. L. Filgueiras. "Crossmedia application design: exploring linear and non-linear narrative abilities". Proceedings 26th Annual ACM International Conference on Design of communication, pp. 225-234. 2008.

[11] C.D. Nguyen, E. Gallagher, A. Read and G.J. De Vreede. "Generating user stories in groups". Lecture Notes Computation Science. Vol. 5784, pp. 357-364. 2009.
[12] R. Akbar, M. Haris and M. Naeem. "Requirement Gathering and Tracking Process for Distributed Agile based Development". Proceedings of the 8th conference on Applied informatics and communications, pp. 429-436. 2008.

[13] D. Connolly, F. Keenan and B. Ryder. "Tag oriented agile requirements identification". Proceedings of Fifteenth IEEE International Conference Working Engineering Computation Systems, pp. 497-498. 2008.

[14] S. Kelly. "Towards an evolutionary framework for agile requirements elicitation". Proceedings of the 2nd ACM SIGCHI symposium on Engineering interactive computing systems, pp. 349352. 2010.

[15] M.C. Leonardi, J. Cesar and P. Leite. "Using Business Rules in E X treme R equirements", pp. 420-435. 2002.

[16] M. Maguire. "Using human factors standards to support user experience and agile design". Lecture Notes Computer Science. Vol. 8009, pp. 185-194. 2013.

[17] A. Singhal. "Development of Agile Security Framework using a Hybrid Technique for Requirements Elicitation”. In Advances in Computing, Communication and Control, Springer, pp. 178-188. 2011.

[18] A. N. Bowers, R. S. Sangwan and C. J. Neill. "Adoption of XP practices in the industry - A survey". Software Process Improvement and Practice. Vol. $12 \mathrm{~N}^{\mathrm{o}} 3$, pp. 283-294. 2007. 